\title{
A NEW IMAGE ENCRYPTION AND DATA HIDING TECHNIQUE USING WAVELET TRANSFORM
}

\author{
M.Dileep ${ }^{1}$, B.Prudhviraj ${ }^{2}$, G.Prasannakumar ${ }^{3}$ \\ ${ }^{1,2,3}$ Assistant Professor, Department of ECE, Vishnu Institute of Technology, AP, India
}

\begin{abstract}
In this paper a new image encryption and decryption is proposed by using wavelet Packet decomposition. Data hiding is also embedding in the encrypted image. In the process of encryption original image is wavelet decomposed; further security can be achieved by rotational transform of the pixels that is the pixels are rotated after decomposing using wavelet transform. The data to be hided is embedded after rotational transform of the image; the resultant image is encrypted image with data hiding .The data recovered from data hided image by using inverse data hiding after the decrypt the image by inverse rotational transform and inverse wavelet decomposition. This encryption and data hiding technique is efficient technique for secure image and data transmission.
\end{abstract}

Keywords: Encryption, Rotational Transformation, Data hiding, and Decryption.

\section{INTRODUCTION}

In recent days secure transmission of image and data is one of the very important criteria in image processing. Cryptology is the secure communication, Image encryption is the process of encoding an image in such a way that only authorized persons can view the image, decryption is the process of decoding the encrypted image, so that the encryption and decryption process are very important in cryptography. Data hiding is the process of hiding a message over an image.

In this paper mainly concentrated on Data hiding over a encrypted image so that more secure data transmission over a image is possible. The original image is encrypted by using wavelet transform and rotational transform, the data to be hided is over an encrypted image. The proposed method uses the rotational transformation and inverse rotational transformation for more secure transmission.

The original image is encrypted by using discrete wavelet decomposition and encrypted key. The wavelet decomposition gives a decomposition matrix by using the given wavelet and a book keeping matrix. The decomposition matrix is column matrix and book keeping matrix is used at the time of reconstruction.

In this paper detailed work is described; the rest of the paper organized as follows, section 2 provides our new encryption process, section 3 presents the rotational transform, section 4 presents the data hiding process, section 5 presents the simulation results, finally conclusions are given in section 6 .

\section{ENCRYPTION PROCESS}

The encryption and data hiding process is shown in block diagram.

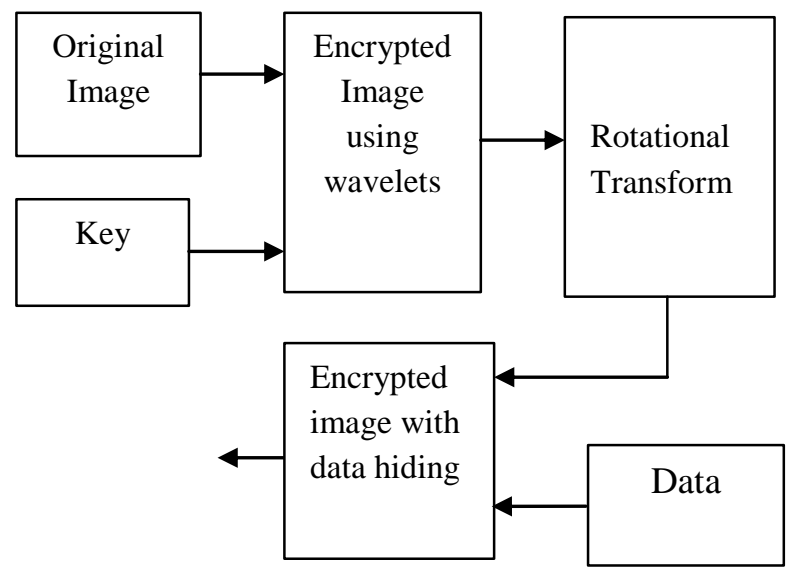

Fig-1: Encryption and data hiding process

The original image is first decomposed into discrete wavelet decomposition; it consists of decomposed matrix and $\mathrm{s}$ matrix which is used as key for decryption process. The decomposed matrix is a single row matrix and that matrix is reshaped into size of the image. The resultant image for other encrypted by using a encryption key resultant encrypted image is rotational transferred that gives the more secured image. The data is hided in encrypted image. 


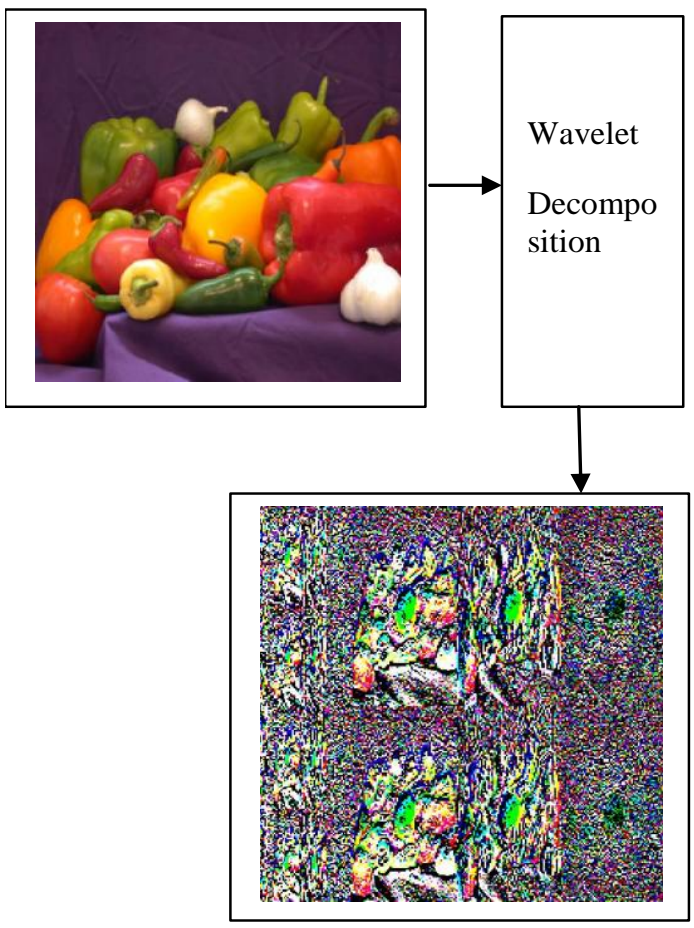

Fig-2: Encrypted using wavelet decomposition

The decomposed image is encrypted using encryption key , for this implemented a reversible equation given by

$$
\begin{aligned}
\text { Encrypted image }= & \text { Decomposed image }-a * k e y \\
& e=d-a * k
\end{aligned}
$$

Where ' $a$ ' is the small value between 0 and 1 ;

By taking the inverse equation we can decrypt the original image.

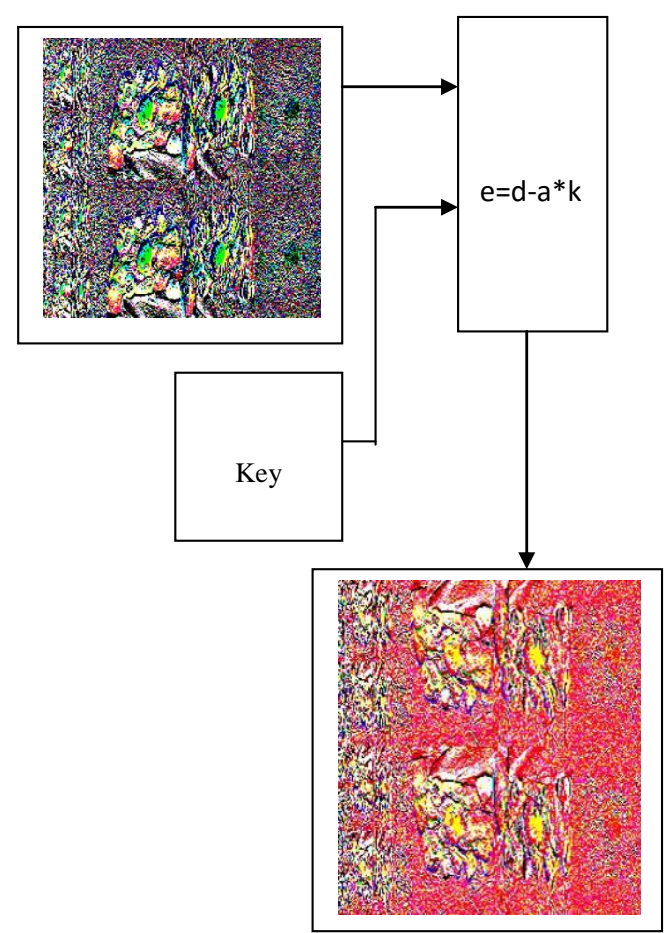

Fig- 3: encrypted image using key
The above figure shows the encrypted image using key, the encrypted key is embedding on the decomposed image by using the equation

\section{ROTATIONAL TRANSFORMATION}

The rotational transformation is a reversible transformation, by this transformation the pixel values are rotated in row wise which gives the more secure encrypted image.

Rotational transformation kernel of size $3 \times 3$ is given by

$$
k=\begin{array}{lll}
0 & 0 & 1 \\
0 & 1 & 0 \\
1 & 0 & 0
\end{array}
$$

Take $\mathrm{x}$ is a $3 \mathrm{x} 3$ matrix

$$
x=\begin{array}{lll}
1 & 2 & 3 \\
4 & 2 & 1 \\
7 & 5 & 2
\end{array}
$$

Multiplication of $\mathrm{x}$ with the kernel $\mathrm{k}$ results

$$
x * k=\begin{array}{rrr}
3 & 2 & 1 \\
1 & 2 & 4 \\
2 & 5 & 7
\end{array}
$$

Similarly for the $5 \times 5$ matrix the rotational transformation kernel is given by

$\mathrm{K}=\begin{array}{lllll}0 & 0 & 0 & 0 & 1 \\ 0 & 0 & 0 & 1 & 0 \\ 0 & 0 & 1 & 0 & 0 \\ 0 & 1 & 0 & 0 & 0 \\ 1 & 0 & 0 & 0 & 0\end{array}$

So by using the similarity of the rotational transformation kernel we can achieve the transformation kernel for the size of the image.

Inverse transformation is achieved by using the same forward transformation kernel at the time of decryption.

\section{DATA HIDING}

The process of hiding a message over an image is the data hiding, first data is converted into numerical values, these numerical values are converted into binary values these binary values replaces the least significant bits of the encrypted image so that more secure data hiding is processed.

Recovering the data from the encrypted image is uses the reverse process of embedding the data, after recovering the data decryption is takes place.

\section{RESULTS}

The simulation results using MATLAB GUI is shown below, we take the peppers image as original image that has to be encrypted 


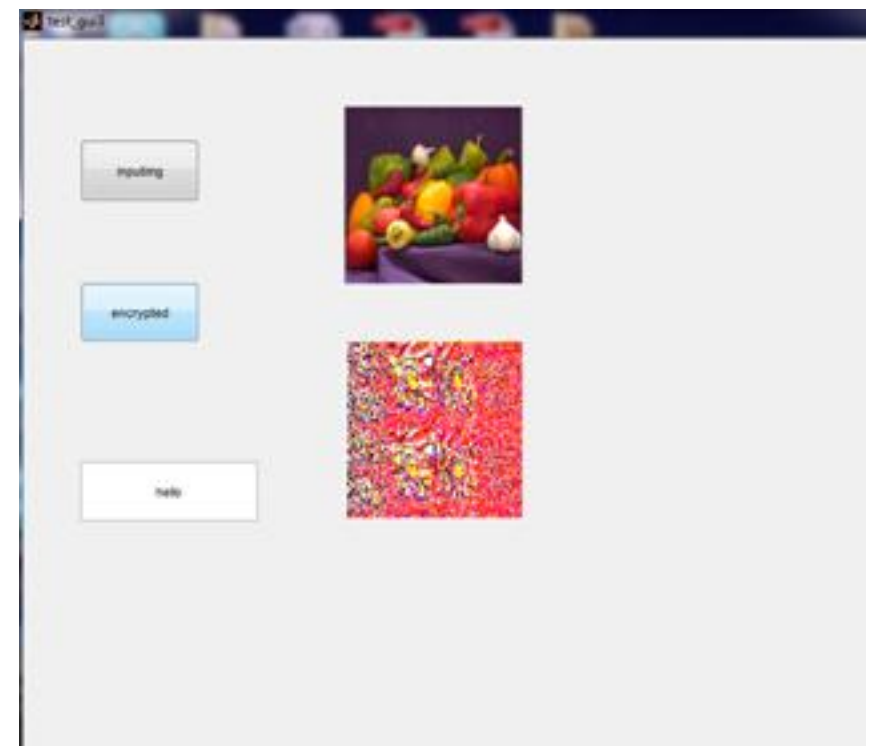

Fig-4: Simulation result of original Image and encrypted image

The peppers image is taken as the original image, it is encrypted using encryption key shown below the original image in figure 4 and text box shows the data to be hided.

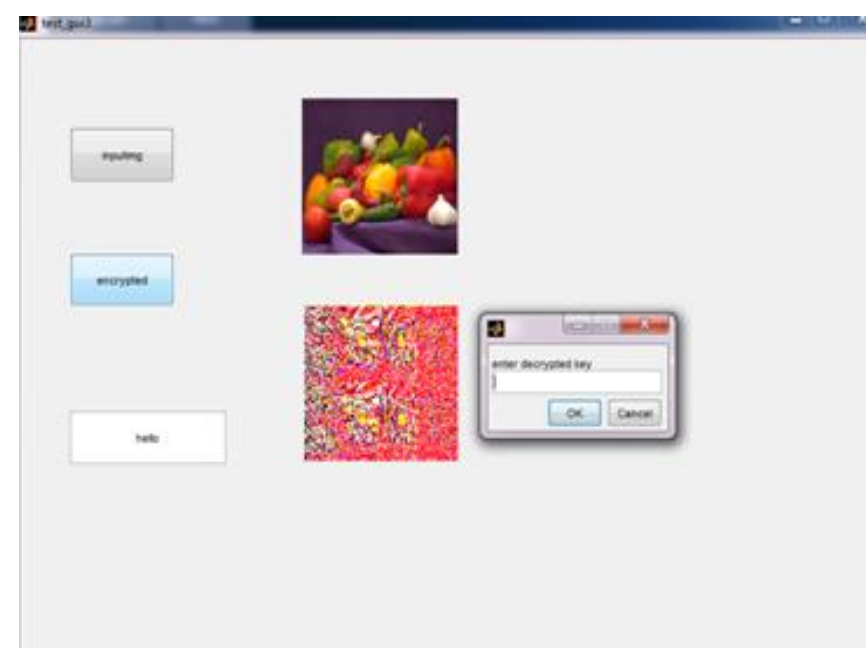

Fig-5: Simulation result of original Image, encrypted image and dialog box for decryption key

After the completion of encryption process decryption to be held by using decryption key that has to be entered through a dialog box shown in figure 5 .

The decryption process is as follows entering the decryption key then encrypted image is decrypted by using key and data is also recovered from the encrypted image shown in text box GUI figure 6

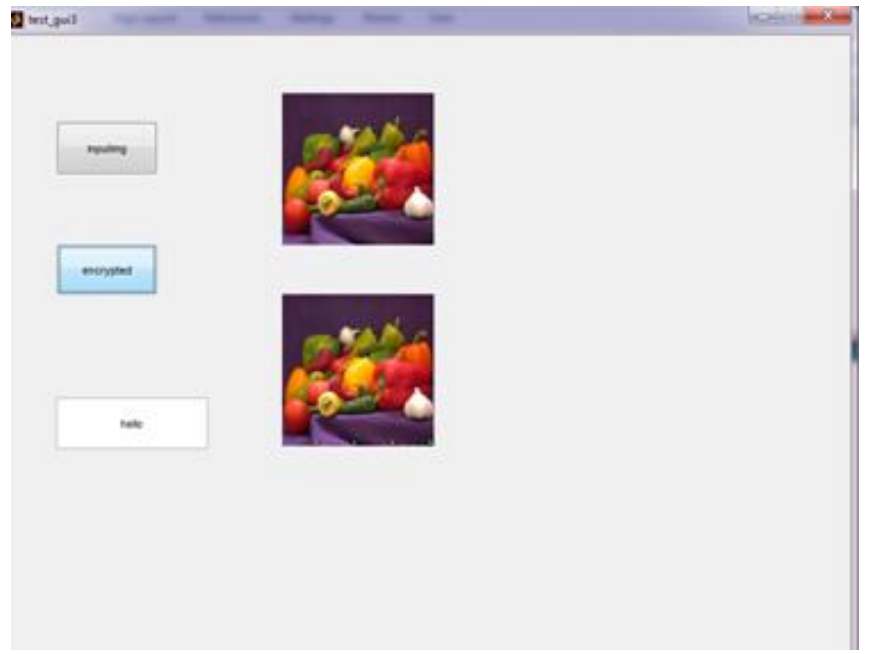

Fig-6: Simulation result of original Image and decrypted image

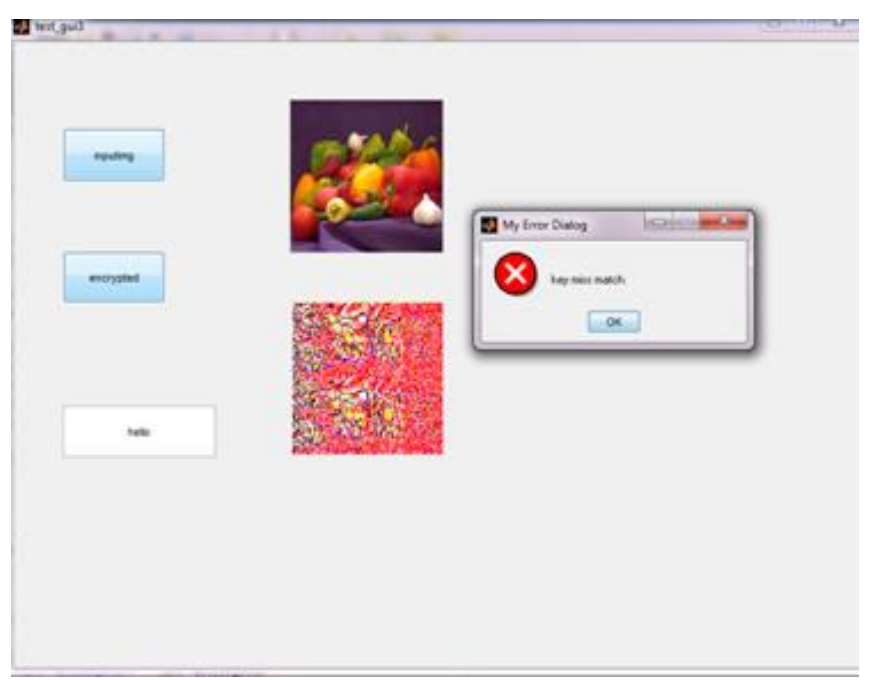

Fig -7: Simulation result when wrong key

When the key entered for decryption process is not matched to key which is for encryption then a dialog box shows that wrong key that is shown in figure 7

\section{CONCLUSIONS}

In this paper we propose a new encryption and decryption algorithm and secure data hiding using wavelet decomposition and rotational transformation. The process of wavelet decomposition and rotational transformation of image gives the more secure encrypted image. Data hiding process in the encrypted image is secure data hiding technique. Recovering the data from the encrypted image uses the reverse process of encryption. The decryption of encrypted image follows the reveres process of first inverse rotational transformation and inverse wavelet decomposition. This new encryption and embedding data technique is more secure and efficient encryption technique.

\section{REFERENCES}

[1]. Zhenxing Qian; Xinpeng Zhang "Reversible Data Hiding in Encrypted Images With Distributed Source Encoding", IEEE Transactions on Circuits and Systems 
for Video Technologyby Year: 2016, Volume: 26, Issue: 4

[2]. Mahita Mahesh, Dhivya Srinivasan,Mila Kankanala,RamachandranAmutha,"Image cryptograph y using Discrete Haar Wavelet Transform and Arnold Cat Map",IEEE Conference Publications Year: 2015

[3]. Arghya Ray, Avishake Ghosh, B.Padhmavathi "A multilayer visual cryptography framework for secured secret messages transmission", Proceedings of 2015 IEEE 9th International Conference on Intelligent Systems and Control (ISCO)

[4]. Md. Rashedul Islam, Ayasha Siddiqa "An efficient filtering based approach improving LSB image steganography using status bit along with AES cryptography', IEEE Conference Informatics, Electronics \& Vision (ICIEV), 2014 International Conference on

[5]. Young-Chang Hou; Shih-Chieh Wei; Chia-Yin Lin,"Random-Grid Based Visual Cryptography Schemes",IEEE Transactions on Circuits and Systems for Video Technology Year: 2014, Volume: 24, Issue: 5

[6]. Kai-Hui Lee; Pei-Ling Chiu "Sharing Visual Secrets in Single Image Random Dot Stereogram's", IEEE Transactions on Image Processing Year: 2014, Volume: 23, Issue: 10

[7]. Dao-Shun Wang, Tao Song, Lin Dong, and ChingNung Yang, "Optimal Contrast Grayscale Visual Cryptography Schemes With Reversing", IEEE Transactions on Information Forensics and Security Year: 2013, Volume: 8, Issue: 12

[8]. Xinpeng Zhang "Separable Reversible Data Hiding in Encrypted Image" IEEE Transactions on Information Forensics and Security, vol. 7, no. 2, April 2012.

[9]. K. Sakthidasan@Sankaran and B. V. Santhosh Krishna "A New Chaotic Algorithm for Image Encryption and Decryption of Digital Color Images" ,International Journal of Information and Education Technology Vol. 1, No. 2, June 2011137

[10].Deergha Rao K. and Gangadhar Ch., (2011) "VLSI Realization of a Secure Cryptosystem for Image Encryption and Decryption" IEEE 2011 International Conference on Communications and Signal Processing (ICCSP).

[11].Xiaojiang Du, Mohsen Guizani, Yang Xiao, HsiaoHwa Chen, "A Routing-Driven Elliptic Curve Cryptography Based Key Management Scheme for Heterogeneous Sensor Networks" IEEE Transactions on Wireless Communications, Vol. 8, no. 3, March 2009

[12].G. Alvarez, L. H.Encinas, and .1.M. Masque, "KnownPlaintext Attack to Two Cryptosystems Based on the BB Equation", IEEE Transactions On Circuits and Systems II: Express Briefs Volume 55, Issue 5, May 2008 Page(s):423 - 426

[13].Rama Murthy N. and Swamy M.N.S, "Cryptographic Applications of BrahmaguptaBhaskara Equation",IEEE Transactions on circuits -I, Regular Papers, Vo1.53, July 2006, pp. 1565-1571.
[14].Yong-Hong Zhang, Bao-Sheng Kang, Xue-Feng Zhang, " Image Encryption Algorithm Based On Chaotic Sequence", Proceedings of the 16th International Conference on Artificial Reality and Telexistence - Workshops(ICAT'06),Hang Zhou, Zheijang, China, pp. 221-223, Nov.2006.

[15].Min Wu, and B. Liu. 2004. Data hiding in digital binary image. IEEE Transactions on Multimedia, Vol. 6, No. 4, August 2004

[16].F. A. P. Petitcolas, R. J. Anderson, and M. G. Kuhn, "Information hiding - a survey," Proc. IEEE, vol. 87, pp. 1062-1078, July 1999

\section{BIOGRAPHIES}

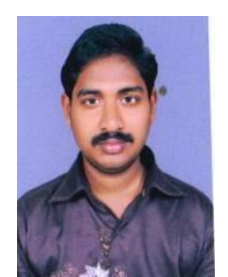

Mr M.Dileep: Assistant. Professor in Vishnu institute of technology. Has six years of teaching Experience, Major working areas are Digital Signal Processing, image processing and Communications Presented research paper in international conferences and published two papers in international journals.

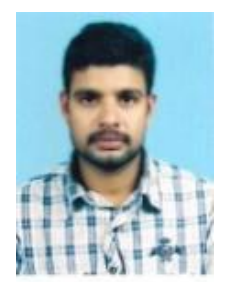

Mr B. Prudhvi Raj: Assistant. Professor in Vishnu institute of technology. Has four years of teaching Experience, Major working areas are Digital Signal Processing and image processing Presented research paper in international conferences and published two papers in international journals.

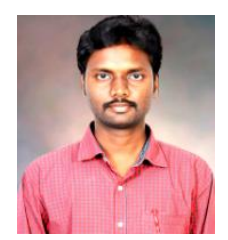

Mr G. Prasanna Kumar: Assistant. Professor in Vishnu institute of technology. Has seven years of teaching Experience, Major working areas are Digital Signal Processing, image processing and VLSI Design Presented research paper in international conferences and published four papers in international journals. 